\title{
Several Asymptotic Bounds on the Balaban Indices of Trees
}

\author{
Bo Deng $\mathbb{D}^{1,}{ }^{1,2,3,4}$ Chengfu Ye, ${ }^{1}$ Weilin Liang, ${ }^{1}$ Yalan Li, ${ }^{5}$ and Xueli Su${ }^{1}$ \\ ${ }^{1}$ School of Mathematics and Statistics, Qinghai Normal University, Xining 810001, China \\ ${ }^{2}$ Academy of Plateau, Science and Sustainability, Xining, Qinghai 810008, China \\ ${ }^{3}$ Key Laboratory of Tibetan Information Processing, Ministry of Education, Xining, Qinghai Province, China \\ ${ }^{4}$ Tibetan Intelligent Information Processing and Machine Translation Key Laboratory, Qinghai 810008, China \\ ${ }^{5}$ School of Computer, Qinghai Normal University, Xining 810001, China
}

Correspondence should be addressed to Bo Deng; dengbo450@163.com

Received 16 August 2020; Revised 30 September 2020; Accepted 21 October 2020; Published 6 November 2020

Academic Editor: Jia-Bao Liu

Copyright $(92020$ Bo Deng et al. This is an open access article distributed under the Creative Commons Attribution License, which permits unrestricted use, distribution, and reproduction in any medium, provided the original work is properly cited.

The Balaban index (also called the $J$ index) of a connected graph $G$ is a distance-based topological index, which has been successfully used in various QSAR and QSPR modeling. Although the index was introduced 30 years ago, there are few results on the asymptotic relations. In this paper, several asymptotic bounds on the Balaban indices of trees with diameters 3 and 4 are shown, respectively.

\section{Introduction}

All graphs considered in this paper are simple and undirected. Let $G$ be a graph with its edge set $E(G)$ and vertex set $V(G)$. We set $|V(G)|=n$ and $|E(G)|=m$. The star of order $n$ is denoted by $S_{n}$. The distance between vertices $u$ and $v$ in $G$ is denoted by $d_{G}(u, v)$, and the sum of the distance between vertex $u$ and each vertex of $G$ is denoted by $\sigma_{G}(u)$, that is, $\sigma_{G}(u)=\sum_{w \in V(G)} d_{G}(u, w)$.

The Balaban index [1] of a connected graph $G$ (or the $J$ index for short) is defined as

$$
J(G)=\frac{m}{\mu+1} \sum_{u v \in E(G)} \frac{1}{\sqrt{\sigma_{G}(u) \sigma_{G}(v)}}
$$

where $\mu$ is the cyclomatic number and $\mu=m-n+1$.

The Balaban index (also called the $J$ index) of a connected graph $G$ is a distance-based topological index, which has been successfully used in various QSAR and QSPR modeling $[2,3]$. Many applications in chemistry can be found in [4-6]. By comparing with the Wiener index regarding alkanes in [7], it was found that the Balaban index reduces the degeneracy of the latter index and provides much higher discriminating ability.
So, the Balaban index is also called the sharpened Wiener index. Some results on the maximal and minimal Balaban index [8-10] have been presented. In [11-14], the asymptotic behaviors of the Balaban indices for various infinite families of graphs are observed.

Until now, there are few results on the asymptotic relations on the Balaban index. In this paper, several asymptotic bounds on the Balaban indices of trees with diameters 3 and 4 are shown, respectively. The two kinds of trees are depicted as follows.

If a tree is with diameter 3 , then this tree can be obtained by attaching some pendent edges to the two end-vertices of one edge. Then, this tree is denoted by $T_{n}(3, a, b)$, see Figure 1 , which has $n$ vertices, diameter 3 , and satisfies that there are $a$ pendent edges attached at one end-vertex of one edge and $b$ pendent edges attached at the other end-vertex of the edge, where $a \geq 1, b \geq 1$, and $a+b=n-2$. The set of this kind of trees is denoted by $\mathscr{T}_{n}(3, a, b)$.

The tree with order $n$ and diameter 4 denoted by $T_{n}^{l}\left(4, a_{1}, a_{2}, \ldots, a\right)$, see Figure 2 , is obtained from a star $S_{l+1}$ by attaching $a_{1}, a_{2}, \ldots, a_{l}$ pendent edges to the $l$ pendent vertices of the star, respectively, where $a_{i} \geq 0,1 \leq i \leq l$. The set of this kind of trees is denoted by $\mathscr{T}_{n}^{l}\left(4, a_{1}, a_{2}, \ldots, a_{l}\right)$. 


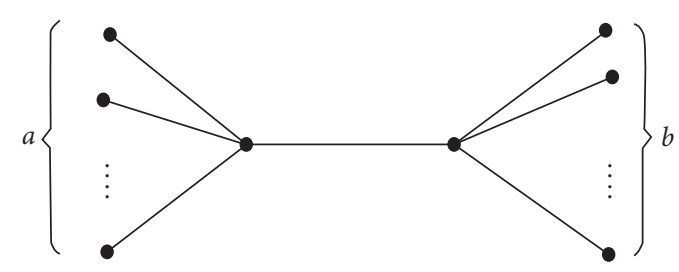

Figure 1: The graph $T_{n}(3, a, b)$.

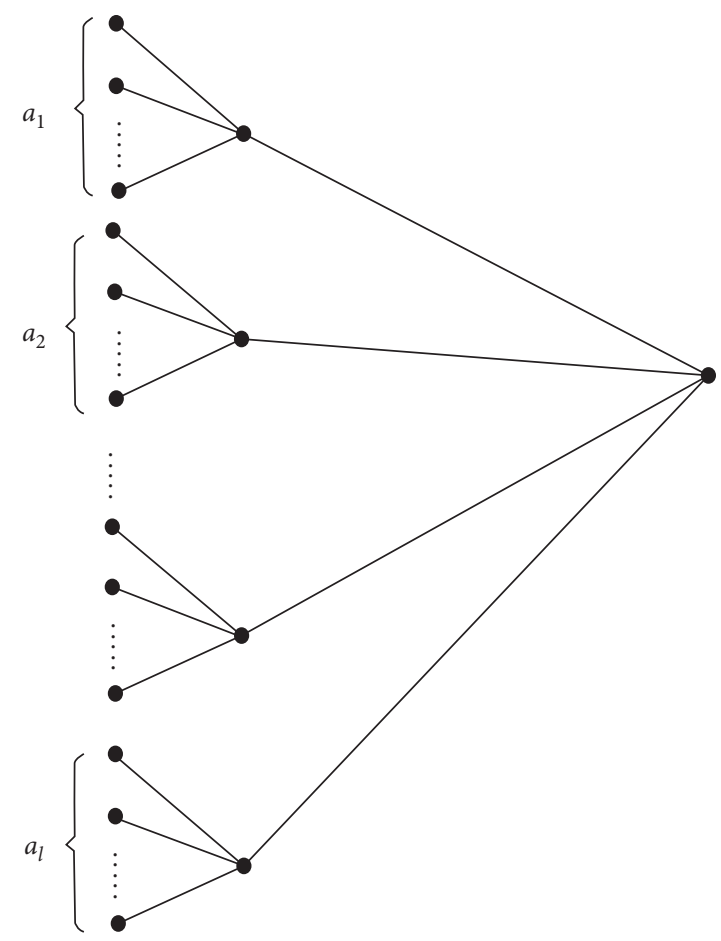

Figure 2: The graph $T_{n}^{l}\left(4, a_{1}, a_{2}, \ldots, a_{l}\right)$.

\section{The Balaban Indices of Trees with Diameter 3}

In this section, the asymptotic bounds on the Balaban indices of trees with diameter 3 will be given.

Theorem 1. For any tree $T_{n}(3, a, b) \in \mathscr{T}_{n}(3, a, b)$, we have

$$
O\left(\frac{2 n}{\sqrt{15}}\right) \leq J\left(T_{n}(3, a, b)\right) \leq O\left(\frac{n}{\sqrt{2}}\right) \text {. }
$$

$$
\begin{aligned}
J\left(T_{n}(3, a, n-a-2)\right)= & (n-1) \\
& \cdot \frac{a}{\sqrt{(3 n-a-5)(2 n-a-3)}}+\frac{1}{\sqrt{(2 n-a-3)(n+a-1)}}+\frac{n-a-2}{\sqrt{(2 n+a-3)(n+a-1)}}
\end{aligned}
$$

Through computation, the derivative of $J\left(T_{n}(3, a, n-\right.$ $a-2)$ ) related to $a$ is 


$$
\begin{aligned}
\frac{\partial J\left(T_{n}(3, a, n-a-2)\right)}{\partial a}= & \frac{n-1}{2} \cdot\left[\frac{a(5 n-2 a-8)}{((3 n-a-5)(2 n-a-3))^{3 / 2}}+\frac{2}{\sqrt{(3 n-a-5)(2 n-a-3)}}+\frac{2+2 a-n}{((2 n-a-3)(n+a-1))^{3 / 2}}\right. \\
& \left.-\frac{2}{\sqrt{(n+a-1)(2 n+a-3)}}+\frac{(2+a-n)(3 n+2 a-4)}{((2 n+a-3)(n+a-1))^{3 / 2}}\right] .
\end{aligned}
$$

Next, the sign of $\left(\partial J\left(T_{n}(3, a, n-a-2)\right)\right) / \partial a$ will be determined. For the above equation, we see that the first two terms are positive, and the last three terms are nonpositive.
Then, we use the sum of the first two terms to divide the sum of the absolute values of the last three terms. So, we get that

$\frac{\left(a(5 n-2 a-8) /((3 n-a-5)(2 n-a-3))^{3 / 2}\right)+(2 /(\sqrt{(3 n-a-5)(2 n-a-3)}))}{n-2 a-2 /((2 n-a-3)(n+a-1))^{3 / 2}+2 / \sqrt{(n+a-1)(2 n+a-3)}+(n-a-2)(3 n+2 a-4) /((2 n+a-3)(n+a-1))^{3 / 2}}$
$=\frac{12 n^{2}-38 n+a(8-5 n)+30}{((3 n-a-5)(2 n-a-3))^{3 / 2}\left(\left((n-2 a-2) /((n+a-1)(2 n-a-3))^{3 / 2}\right)+(2 / \sqrt{(n+a-1)(2 n+a-3)})+\left((n-a-2)(3 n+2 a-4) /((2 n+a-3)(n+a-1))^{3 / 2}\right)\right)}$

Denote the above fraction by $f(n, a)$. Observing this quotient, its value is equal to 1 if $n=2 k+2$ and $a=k$ for $k=2,3, \ldots,[n-2 / 2]$. In this case, it means that $T_{n}(3, a, n-$ $a-2)$ attains the minimum value. Otherwise, its value is less than 1 as $n$ tends to infinity, that is,

$$
\lim _{n \longrightarrow \infty} f(n, a)=\frac{4}{7 \sqrt{3}}<1 .
$$

Thus, the derivative of $J\left(T_{n}(3, a, n-a-2)\right)$ related to $a$ is negative as $n$ is big enough. In this case, $J\left(T_{n}(3, a, n-\right.$ $a-2)$ ) increases along with parameter $a$ decreasing. Since $1 \leq a \leq\lfloor(n-2) / 2\rfloor$, we see that

$$
\begin{aligned}
& J\left(T_{n}(3, a, n-a-2)\right) \leq J\left(T_{n}(3,1, n-3)\right), \\
& J\left(T_{n}(3, a, n-a-2)\right) \geq J\left(T_{n}(3,\lfloor(n-2) / 2\rfloor,\lceil(n-2) / 2\rceil)\right) .
\end{aligned}
$$

By calculation, we get

$$
\begin{array}{r}
J\left(T_{n}(3,1, n-3)\right)=O\left(\frac{n}{\sqrt{2}}\right), \\
J\left(\left(T_{n}(3,\lfloor(n-2) / 2\rfloor,\lceil(n-2) / 2\rceil)\right)=O\left(\frac{2 n}{\sqrt{15}}\right)\right) .
\end{array}
$$

Hence, we obtain

$$
O\left(\frac{2 n}{\sqrt{15}}\right) \leq J\left(T_{n}(3, a, b)\right) \leq O\left(\frac{n}{\sqrt{2}}\right)
$$

\section{The Balaban Indices of Trees with Diameter 4}

In this section, we present some asymptotic bounds for the Balaban indices of trees with diameter 4 .

Theorem 2. For any tree $T_{n}^{l}\left(4, a_{1}, a_{2}, \ldots, a_{l}\right) \in$ $\mathscr{T}_{n}^{l}\left(4, a_{1}, a_{2}, \ldots, a_{l}\right)$, we have

$$
J\left(4, a_{1}, a_{2}, \ldots, a_{l}\right) \leq O\left(\frac{n}{\sqrt{2}}\right) .
$$

Proof. The Balaban index of a tree $T_{n}^{l}\left(4, a_{1}, a_{2}, \ldots, a_{l}\right)$ is as follows:

$$
\begin{aligned}
J\left(T_{n}^{l}\left(4, a_{1}, a_{2}, \ldots, a_{l}\right)\right)= & (n-1) \\
& \cdot\left[\sum_{i=1}^{l} \frac{a_{i}}{\sqrt{\left(4 n-2 a_{i}-l-6\right)\left(3 n-2 a_{i}-l-4\right)}}+\sum_{i=1}^{l} \frac{1}{\sqrt{(2 n-l-2)\left(3 n-2 a_{i}-l-4\right)}}\right] .
\end{aligned}
$$
Then,

Suppose $1 \leq a_{1} \leq a_{2}$, and let $a_{1}=a$ and $c=n-a-a_{2}$. 


$$
J\left(T_{n}^{l}\left(4, a_{1}, a_{2}, \ldots, a_{l}\right)\right)=(n-1) \cdot\left[\begin{array}{c}
\frac{a}{\sqrt{(4 n-2 a-l-6)(3 n-2 a-l-4)}} \\
+\frac{a_{i}}{\sqrt{(2 n+2 a+2 c-l-6)(n+2 a+2 c-l-4)}} \\
+\sum_{i=3}^{l} \frac{1}{\sqrt{\left(4 n-2 a_{i}-l-6\right)\left(3 n-2 a_{i}-l-4\right)}} \\
+\frac{1}{\sqrt{(2 n-l-2)(3 n-2 a-l-4)}}+\frac{1}{\sqrt{(2 n-l-2)(n+2 a+2 c-l-4)}} \\
+\sum_{i=3}^{l} \frac{1}{\sqrt{(2 n-l-2)\left(3 n-2 a_{i}-l-4\right)}}
\end{array}\right]
$$

And we get the derivative of $J\left(T_{n}^{l}\left(4, a, a_{2}, \ldots, a_{l}\right)\right)$ related to $a$ as follows:

$$
\begin{aligned}
& \frac{-2-l+2 n}{((-2-l+2 n)(-4-2 a-l+3 n))^{3 / 2}} \\
& +\frac{1}{((-4-2 a-l+3 n)(-6-2 a-l+4 n))^{1 / 2}} \\
& \frac{\partial J\left(T_{n}^{l}\left(4, a, a_{2}, \cdots, a_{l}\right)\right)}{\partial a}=(n-1) . \\
& -\frac{-2-l+2 n}{((-2-l+2 n)(-4-l+n+2 a+2 c))^{3 / 2}} \\
& -\frac{1}{((-4-l+n+2 a+2 c)(-6-l+2 n+2 a+2 c))^{1 / 2}} \\
& +\frac{a(-20-8 a-4 l+14 n)}{2((-4-2 a-l+3 n)(-6-2 a-l+4 n))^{3 / 2}} \\
& {\left[-\frac{(-a-c+n)(-20-4 l+6 n+8 a+8 c)}{2((-4-l+n+2 a+2 c)(-6-l+2 n+2 a+2 c))^{3 / 2}}\right]}
\end{aligned}
$$

The sum of positive terms above is denoted by $S^{+}(n, a, l)$, i.e.,

$$
S^{+}(n, a, l)=(n-1) \cdot\left[\begin{array}{c}
\frac{-2-l+2 n}{((-2-l+2 n)(-4-2 a-l+3 n))^{3 / 2}} \\
+\frac{1}{((-4-2 a-l+3 n)(-6-2 a-l+4 n))^{1 / 2}} \\
+\frac{a(-20-8 a-4 l+14 n)}{2((-4-2 a-l+3 n)(-6-2 a-l+4 n))^{3 / 2}}
\end{array}\right] .
$$

And the sum of absolute values of negative terms above is denoted by $S^{-}(n, a, l)$, i.e., 


$$
S^{-}(n, a, l)=(n-1) \cdot\left[\begin{array}{c}
\frac{-2-l+2 n}{((-2-l+2 n)(-4-l+n+2 a+2 c))^{3 / 2}} \\
+\frac{1}{((-4-l+n+2 a+2 c)(-6-l+2 n+2 a+2 c))^{1 / 2}} \\
+\frac{(-a-c+n)(-20-4 l+6 n+8 a+8 c)}{2((-4-l+n+2 a+2 c)(-6-l+2 n+2 a+2 c))^{3 / 2}}
\end{array}\right] .
$$

Then, we use $S^{+}(n, a, l)$ to divide $S^{-}(n, a, l)$, and we get

$$
\lim _{n \rightarrow \infty} \frac{S^{-}(n, a, l)}{S^{+}(n, a, l)}=\frac{5}{2^{3 / 2}}>1 \text {. }
$$

So, the derivative of $J\left(T_{n}^{l}\left(4, a_{1}, a_{2}, \ldots, a_{l}\right)\right)$ related to $a_{1}$ is less than 0 as $n$ is big enough. It means that the corresponding Balaban index increases along with the number of pendent edges $a_{1}$ decreasing and the number of pendent edges $a_{2}$ increasing, i.e.,

$$
J\left(T_{n}^{l}\left(4, a_{1}, a_{2}, \ldots, a_{l}\right)\right) \leq J\left(T_{n}^{l}\left(4, a_{1}-1, a_{2}+1, \ldots, a_{l}\right)\right) .
$$

Analogously, we obtain

$$
\begin{aligned}
J\left(T_{n}^{l}\left(4, a_{1}, a_{2}, \ldots, a_{l}\right)\right) & \leq J\left(T_{n}^{l}\left(4,1, a_{1}+a_{2}+a_{3}+\cdots a_{l}-1,0, \ldots, 0\right)\right) \\
& =J\left(T_{n}^{l}(4,1, n-l-2,0, \ldots, 0)\right) .
\end{aligned}
$$

For the above tree $T_{n}^{l}(4,1, n-l-2,0, \ldots, 0)$, it can be seen that there are only two numbers, i.e., 1 and $n-l-2$, as the numbers of pendent edges, respectively, attach to two pendent vertices of a star $S_{l+1}$. Thus, it is easy to check that

$$
J\left(T_{n}^{l}(4,1, n-l-2,0, \ldots, 0)\right)=O\left(\frac{n}{\sqrt{2}}\right) .
$$

Hence,

$$
J\left(T_{n}^{l}\left(4, a_{1}, a_{2}, \ldots, a_{l}\right)\right) \leq O\left(\frac{n}{\sqrt{2}}\right) .
$$

On the contrary, the asymptotically tight lower bound of such a tree is not easy to be given due to the determination of parameter $l$, but we find that, in $\mathscr{T}_{n}^{l}\left(4, a_{1}, a_{2}, \ldots, a_{l}\right)$, the tree attained the asymptotically tight lower bound which possesses a property satisfying $\left|a_{i}-a_{j}\right| \leq 1$ for $1 \leq i \neq j \leq l$. In case of $l=2$, we obtain the following result.

Theorem 3. For any tree $T_{n}^{2}\left(4, a_{1}, a_{2}\right) \in \mathscr{T}_{n}^{2}\left(4, a_{1}, a_{2}\right)$, where $1 \leq a_{1} \leq a_{2}$, we have

$$
J\left(T_{n}^{2}\left(4, a_{1}, a_{2}\right)\right) \geq O\left(\frac{n}{\sqrt{6}}\right) .
$$

Proof. From the proof of Theorem 2, we see that the derivative of $J\left(T_{n}^{2}\left(4, a_{1}, a_{2}\right)\right)$ related to $a_{1}$ is less than 0 as $n$ is big enough. Thus, if $a_{1}=\lfloor n-3 / 2\rfloor, a_{2}=\lceil n-3 / 2\rceil$, then

$$
J\left(T_{n}^{2}\left(4, a_{1}, a_{2}\right)\right) \geq J\left(T_{n}^{2}\left(4,\left\lfloor\frac{n-3}{2}\right\rfloor,\left\lceil\frac{n-3}{2}\right\rceil\right)\right) .
$$

Note that

$$
J\left(T_{n}^{2}\left(4,\left\lfloor\frac{n-3}{2}\right\rfloor,\left\lceil\frac{n-3}{2}\right\rceil\right)\right)=O\left(\frac{n}{\sqrt{6}}\right) .
$$

Thus,

$$
J\left(T_{n}^{2}\left(4, a_{1}, a_{2}\right)\right) \geq O\left(\frac{n}{\sqrt{6}}\right) .
$$

\section{Data Availability}

The data used to support the findings of this study are included within the article.

\section{Conflicts of Interest}

The authors declare that they have no conflicts of interest.

\section{Acknowledgments}

This research was supported by the NSFQH (no. 2018-ZJ925Q) and NSFC (no. 11701311).

\section{References}

[1] A. T. Balaban, "Highly discriminating distance-based topological index," Chemical Physics Letters, vol. 89, no. 5, pp. 399-404, 1982.

[2] J. Devillers and A. T. Balaban, Topological Indices and Related Descriptors in QSAR and QSPR, Gordon \& Breach, Amsterdam, The Netherlands, 1999.

[3] R. Todeschini and V. Consonni, Handbook of Molecular Descriptors, Wiley, Weinheim, Germany, 2000.

[4] A. T. Balaban, P. V. Khadikar, C. T. Supuran, A. Thakur, and M. Thakur, "Study on supramolecular complexing ability visà-vis estimation of $\mathrm{pKa}$ of substituted sulfonamides: 
dominating role of Balaban index (j)," Bioorganic \& Medicinal Chemistry Letters, vol. 15, no. 17, pp. 3966-3973, 2005.

[5] G. Grassy, B. Calas, A. Yasri et al., "Computer-assisted rational design of immunosuppressive compounds," Nature Biotechnology, vol. 16, no. 8, pp. 748-752, 1998.

[6] P. V. Khadikar, C. T. Supuran, A. Thakur, and M. Thakur, "QSAR study on benzene-sulphonamide carbonic anhydrase inhibitors: topological approach using balaban index," Bioorganic \& Medicinal Chemistry, vol. 12, pp. 789-793, 2004.

[7] A. T. Balaban, "A comparison between various topological indices, particularly between the index $J$ and Wiener index ,W, " in Topology in Chemistry Discrete Mathematics of Molecules, D. H. Rouvray and R. B. King, Eds., Horwood, Chichester, England, 2002.

[8] H. Dong and X. Guo, "Character of graphs with extremal balaban index," MATCH Communications in Mathematical and in Computer Chemistry, vol. 63, pp. 799-812, 2010.

[9] L. Sun, "Bounds on the balaban index of trees," MATCH Communications in Mathematical and in Computer Chemistry, vol. 63, pp. 813-818, 2010.

[10] B. Zhou and N. TrinajstiSc, "Bounds on the balaban index," Croatica Chemica Acta, vol. 81, pp. 319-323, 2008.

[11] A. T. Balaban, N. Ionescu-Pallas, and T. S. Balaban, "Asymptotic values of topological indices $j$ and $j$ ' (average distance sum connectivities) for infinite cyclic and acyclic graphs," MATCH Communications in Mathematical and in Computer Chemistry, vol. 17, pp. 121-146, 1985.

[12] M. Knor, J. Kranjc, R. Škrekovski, and A. Tepeh, "A search for the minimum value of balaban index," Applied Mathematics and Computation, vol. 286, pp. 301-310, 2016.

[13] M. Knor, J. Kranjc, R. Krekovski, and A. Tepeh, "A note on accumulation points of balaban index," MATCH Communications in Mathematical and in Computer Chemistry, vol. 78, pp. 163-168, 2017.

[14] M. Knor, J. Kranjc, R. Škrekovski, and A. Tepeh, "On the minimum value of sum-balaban index," Applied Mathematics and Computation, vol. 303, pp. 203-210, 2017. 